\title{
Telephone Interview for Cognitive Status (TICS): Italian adaptation, psychometrics and diagnostics
}

\author{
Edoardo Nicolò Aiello ${ }^{1}$ (D) Antonella Esposito ${ }^{2} \cdot$ Ilaria Giannone $^{2} \cdot$ Lorenzo Diana $^{1} \cdot$ Ildebrando Appollonio $^{3}$. \\ Nadia Bolognini ${ }^{2,4}$
}

Received: 13 September 2021 / Accepted: 2 November 2021 / Published online: 18 November 2021

(c) Fondazione Società Italiana di Neurologia 2021

\begin{abstract}
Background Telephone-based cognitive screening (TBCS) is crucial to telehealth care of neurological patients, prevention campaigns, and epidemiological studies on cognitive impairment. The Telephone Interview for Cognitive Status (TICS) is one of the most widespread and psychometrically/diagnostically sound TBCS test, with several versions developed worldwide (e.g., with and without a delayed recall item). In Italy, only attempts of adaptation and preliminary evidence of its statistical features have been provided so far. This study thus aimed at (1) developing an Italian version of the TICS and assessing its (2) psychometric and (3) diagnostic properties.

Methods A back-translated and culturally adapted version of the TICS was developed. Three-hundred and sixty-five healthy individuals from different regions of Italy (147 males, 216 females; age: $53.2 \pm 16$ years; education: $13 \pm 4.5$ years) were administered the TICS and the Italian telephone-based Mini-Mental State Examination (Itel-MMSE). Validity was tested by convergence and at the structure level, whereas reliability as internal consistency, test-retest, and inter-rater. Diagnostic accuracy, item difficulty, and discrimination were also examined.

Results The TICS featured a single component and its score converged with that of the Itel-MMSE $\left(r_{s}=.37\right)$. Reliability was excellent as inter-rater (ICC $=.94)$, good as test-retest $(\mathrm{ICC}=.78)$, and acceptable as internal consistency (Cronbach's $\alpha=.63$ ). Accuracy was high as tested against the Itel-MMSE (AUC $=.83$ ) and did not improve when adding the delayed recall. Backward subtraction was the most difficult and discriminative task.

Discussion The Italian TICS is a valid, reliable, and diagnostically accurate TBCS test. The original format of the TICS can be thus adopted in both clinical and research settings.
\end{abstract}

Keywords Telephone Interview for Cognitive Status · Telephone-based · Cognitive screening · Psychometrics · Epidemiology

Ildebrando Appollonio and Nadia Bolognini contributed equally to this work.

Edoardo Nicolò Aiello

e.aiello5@campus.unimib.it

Antonella Esposito

a.esposito32@campus.unimib.it

Ilaria Giannone

i.giannone2@campus.unimib.it

Lorenzo Diana

1.diana1@ campus.unimib.it

Ildebrando Appollonio

illdebrando.appollonio@unimib.it

Nadia Bolognini

nadia.bolognini@unimib.it
1 PhD Program in Neuroscience, School of Medicine and Surgery, University of Milano-Bicocca, Monza, Italy

2 Department of Psychology, University of Milano-Bicocca, Milan, Italy

3 Neurology Section, School of Medicine and Surgery, University of Milano-Bicocca, Monza, Italy

4 Neuropsychological Laboratory, IRCCS Istituto Auxologico Italiano, Milan, Italy 


\section{Introduction}

Telephone-based cognitive screening (TBCS) is crucial to both clinical and experimental telemedicine. Indeed, besides allowing clinicians to deliver first-level assessments to individuals with poor access to de visu healthcare services [1, 2], TBCS eases the implementation of large-scale epidemiological studies [3] and prevention campaigns [4]. In this regard, the ongoing COVID-19 pandemic has accelerated the recourse to TBCS tools, increasing the need of standardized instruments for the remote assessment of the subjective and objective cognitive complaints/failures in the general and elderly populations during restrictions and lockdowns [5-7].

Moreover, when compared to videoconference-based approaches, TBCS more easily allows reaching underserved or elder populations since it requires minimal technological support and expertise [8,9].

Among TBCS instruments, the Telephone Interview for Cognitive Status (TICS) [10] is one of the most widespread and statistically sound test, assessing both instrumental domains (orientation, language, and memory) and attentive/ executive functions [11]. Its applicability in research settings and clinical usability in different neurological populations has been extensively demonstrated $[8,12,13]$.

Several versions of the TICS have been developed worldwide, differing, for instance, for the presence or absence of a delayed recall task [14], although little consensus has been reached as for its optimal format $[13,15]$.

In Italy, the original TICS version [10] has been shown to be administrable face-to-face [16]; as for its remote use, an attempt to its adaptation with promising, albeit preliminary, evidence of its psychometric and diagnostic goodness dates back to 2006 [17]. However, a comprehensive, up-todate standardization study for the Italian TICS has not been provided yet.

With these premises, the present study aimed at (1) developing a culture- and language-specific Italian version of the TICS while assessing its (2) psychometric and (3) diagnostic properties.

\section{Methods}

\section{Participants}

Three-hundred and sixty-five participants from different Italian regions were recruited (see Tables 1 and 2). Demographic and occupational data were collected (see Table 1). Occupational status was codified as white- vs. blue-collar based on the nature of working activities carried out the most during the individual lifespan (i.e., primarily manual vs. clerical job activities). Exclusion criteria were as follows: (1) having received a clinical diagnosis of neurological or psychiatric diseases; (2) severe internal-medical conditions and organ/system failures; (3) non-compensated metabolic disorders; and (4) uncorrected hearing deficits. Participants were recruited between 2020 and 2021; some of them were personal acquaintances of researchers from the University

Table 2 Demographic and cognitive data

\begin{tabular}{lll}
\hline $\boldsymbol{N}$ & & 365 \\
Age (years) & & $53.16 \pm 16.03(18-89)$ \\
Sex (M/F) & & $147 / 218$ \\
Education (years) & & $13.01 \pm 4.46(0-26)$ \\
Italian regions $(\boldsymbol{N})$ & North Italy & 271 \\
& Center Italy & 11 \\
& South Italy & 83 \\
Occupation $(\boldsymbol{N})$ & White-collar & 159 \\
& Blue-collar & 206 \\
Itel-MMSE & & $21.47 \pm 1.05(14-22)$ \\
TICS & Total score (1-41) & $34.81 \pm 3.06(22-41)$ \\
& Orientation (0-12) & $11.74 \pm 0.55(9-12)$ \\
& Language (1-8) & $7.72 \pm 0.53(6-8)$ \\
& Attention and & $8.36 \pm 1.35(2-9)$ \\
& executive functioning & \\
& (0-9) & $6.99 \pm 1.97(1-12)$ \\
\hline
\end{tabular}

$N$, number of participants; $M$, male; $F$, female; Itel-MMSE, Italian telephone-based Mini-Mental State Examination; TICS, Telephone Interview for Cognitive Status
Table 1 Sample stratification for age, education, and sex

\begin{tabular}{|c|c|c|c|c|c|c|c|}
\hline \multirow[t]{2}{*}{ Education } & \multicolumn{7}{|c|}{ Age $(M / F)$} \\
\hline & $35 \leq$ & $36-50$ & $51-65$ & $66-75$ & $76-80$ & $\geq 86$ & Total \\
\hline $5 \leq$ & $0 / 0$ & $0 / 0$ & $0 / 1$ & $2 / 6$ & $5 / 11$ & $0 / 2$ & $7 / 20$ \\
\hline $6-8$ & $1 / 1$ & $2 / 12$ & $13 / 18$ & $4 / 7$ & $3 / 6$ & $1 / 0$ & $24 / 44$ \\
\hline $9-13$ & $17 / 7$ & $7 / 21$ & $33 / 42$ & $6 / 4$ & $2 / 3$ & $1 / 0$ & $66 / 77$ \\
\hline $14-16$ & $6 / 8$ & $2 / 5$ & $1 / 4$ & $0 / 0$ & $2 / 0$ & $0 / 0$ & $11 / 17$ \\
\hline$\geq 17$ & $11 / 18$ & $7 / 7$ & $19 / 29$ & $1 / 3$ & $1 / 3$ & $0 / 0$ & $39 / 60$ \\
\hline Total & $35 / 34$ & $18 / 45$ & $66 / 94$ & $13 / 20$ & $13 / 23$ & $2 / 2$ & $147 / 218$ \\
\hline
\end{tabular}

Cells show male/female (M/F) ratio for each co-occurrence 
of Milano-Bicocca, others were recruited via word-to-mouth advertising. The study was approved by the Ethics Committee of the University of Milano-Bicocca. Participants provided their informed consent to their participation to the study.

\section{Materials}

A back-translated version of the original, English TICS developed by Brandt et al. [10] was adopted. Culture-specific items were adapted according to Ferrucci et al.'s [16] guidelines. No disagreements on linguistic aspects emerged among the authors, while minor discrepancies on cultural adjustments were solved throughout discussion.

The original TICS score [10] ranges from 1 to 41 and comprises 11 items assessing orientation (personal, temporal, and spatial; score range: 0-12), attention and executive functioning (backward counting, backward calculation, abstraction; range: 0-9), language (naming to description, sentence repetition, and oral comprehension; range: 1-8), and memory (immediate recall, semantic memory; range: $0-12$ ). An off-label delayed recall subtest (DR) of the 10 -word list was additionally administered to $N=152$ participants as the last task. The total TICS score comprising DR thus ranges $1-51$.

Participants were also administered the Italian telephonebased Mini-Mental State Examination (Itel-MMSE) [18], a TBCS test whose validity and reliability has been previously demonstrated [19].

\section{Procedures}

Call quality was tested via an in-depth sound-check from both the examiner and the examinee standpoints (see Supplementary Material 1). The examinee was preliminarily introduced to those actions required to execute tasks during the assessment. An informer was required to (1) ensure about the absence of facilitations within the setting and (2) confirm address information provided by the examinee (as needed to test spatial orientation). Two raters independently scored $N=57$ protocols to test inter-rater agreement. Seventy-seven participants were followed up at a 30-day distance to assess test-retest consistency.

\section{Statistics}

Analyses were performed via R 4.1.0 [20], SPSS 27 [21], and Stata 16 [22].

Minimum sample dimension was estimated at $N=318$ based on a correlational model with a small-to-medium effect size $(\rho=0.2 ; 1-\beta=0.95$; two-tailed $\alpha=0.05)$ via the $\mathrm{R}$ package $p w r$ [23].
Skewness and kurtosis values were judged as indexing non-normality if $\geq|1|$ and $|3|$, respectively [24]. As cognitive measures proved not to distribute normally, associations of interest were tested through non-parametric techniques. More specifically, the relation between cognitive measures, as well as that between cognitive measures and age and education, was tested through Spearman's coefficient. Consistently, the interplay between sex and TICS measures was tested via Mann-Whitney tests. Bonferroni corrections for multiple comparisons were performed when adequate.

Factorial structure was investigated through principal component analyses. Internal consistency was tested via Cronbach's $\alpha$, computed on dichotomous items via the $\mathrm{R}$ package ltm [25]. Test-retest and inter-rater reliability were assessed via intra-class correlations.

Item difficulty and discrimination were examined by means of an item response theory two-parameter logistic model [26] run via the $\mathrm{R}$ package mirt [27]. Canonical difficulty was judged for values ranging from -4 to $+4[28$, 29]. With regard to discrimination, items could be classified as "discriminative" $(\geq 1.5)$ or "highly discriminative" $(\geq 1.7)$ [29].

Receiver-operating characteristics analyses were run to test diagnostic accuracy. A performance below vs. above the 5th percentile on the Itel-MMSE was addressed as a proxy gold standard.

\section{Results}

Mean age of participants was $53.16 \pm 16.03$ years (range: $18-89$ years), whereas mean education was $13.01 \pm 4.46$ years (range: $0-26$ years); 147 participants were males, 218 were females. The majority of participants came from Northern Italy $(N=271)$, whereas 11 were from Center and 83 from Southern Italy. One-hundred and fiftynine participants were classified as white-collar, whereas 206 as blue-collar. Cognitive scores are also summarized in Table 2.

All TICS measures, except for orientation sub-scores, were inversely related to age $\left(-0.36 \leq r_{s-365} \leq-0.12\right.$; $p \leq 0.05)$ and positively associated to education $\left(0.13 \leq r_{s-365} \leq 0.40 ; p \leq 0.05\right)$; no sex differences were found $(p \geq 0.24)$.

Total scores on the TICS and Itel-MMSE proved to significantly converge $\left(r_{s-365}=0.37 ; p<0.001\right)$. Itel-MMSE scores were associated with all TICS sub-scores, but the strongest correlation was found with the orientation subtest (see Table 3). All TICS sub-scores were internally related at $\alpha_{\text {adjusted }}=0.008\left(0.18 \leq r_{s-365} \leq 0.28 ; p \leq 0.001\right)$ except for the orientation subscale, which was associated with attentive/executive sub-scores only $(p<0.001)$. TICS total scores correlated with all of its sub-scores at 
Table 3 Spearman's coefficients between the Itel-MMSE and TICS scores

\begin{tabular}{llllll}
\hline & TICS & TICS-O & TICS-L & TICS-AEF & TICS-M \\
\hline Itel-MMSE & $.37^{*}$ & $.56^{*}$ & $.12^{\dagger}$ & $.30^{*}$ & $.2^{*}$ \\
\hline
\end{tabular}

${ }^{\dagger} p=.017 ;{ }^{*} p<.001 ; O$, orientation; $L$, language; $A E F$, attention and executive functioning; $M$, memory

$\alpha_{\text {adjusted }}=0.013$ (orientation: $r_{s}=0.31$; attention/executive functioning: $r_{s}=0.59$; memory: $r_{s}=0.86$; language: $r_{s}=0.39 ;$ all $N \mathrm{~s}=365$ and $\left.p \mathrm{~s}<0.001\right)$. Consistently, DR items proved to be associated with the memory subtest $\left(r_{s-152}=0.73 ; p<0.001\right)$ and the total score $\left(r_{s-152}=0.61\right.$; $p<0.001)$.

A clear mono-component structure was detected (here denominated "global cognition/cognitive efficiency") that explained $18.84 \%$ of variance, with moderate-to-high saturations $(0.31 \leq r \leq 0.68)$, except for repetition, personal, and temporal orientation items $(r<0.3)$. Reliability was excellent as inter-rater ( $\mathrm{ICC}=0.94)$, good as test-retest $(\mathrm{ICC}=0.78)$, and internally acceptable (Cronbach's $\alpha=0.63)$.

A summary of item difficulty and discrimination values is reported in Table 4. Overall, TICS items showed moderate-to-high difficulty, with backward subtraction task yielding the highest difficulty. Backward subtraction items also proved to be the most discriminative.

The TICS proved to be highly accurate in discriminating between those performing below vs. above the 5th percentile of the Itel-MMSE (see Fig. 1); similar findings were obtained when comparing the TICS with vs. without DR (see Fig. 2), with the former being slightly more accurate than the latter $\left(\chi^{2}(1)=3.84 ; p=0.050\right)$.

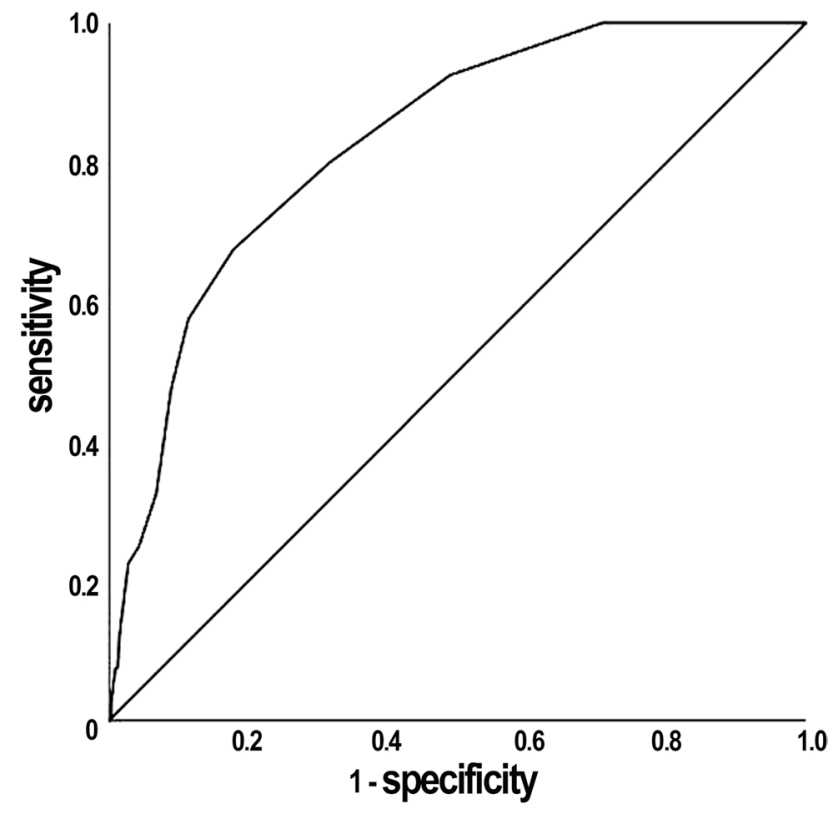

Fig. 1 Receiver-operating characteristics (ROC) curve for the TICS against the Itel-MMSE. The reference measures were a performance above vs. below the 5 th percentile on the Itel-MMSE. AUC $=.83$, $\mathrm{SE}=.03,95 \%$ CI $[.77, .89]$

\section{Discussion}

The present work provides Italian clinicians/researchers with updated evidence supporting the validity, reliability, and diagnostic soundness of a back-translated and culturally adapted Italian version of the TICS. Its adoption is indicated for epidemiological studies [3] and clinical trials [30], as well as for telemedicine practice, opening up to easier
Table 4 Item difficulty and discrimination for the TICS

\begin{tabular}{llll}
\hline Sub-test & Items $(N)$ & Difficulty & Discrimination \\
\hline Orientation & Personal (1)^ & $4.28^{\dagger}$ & .02 \\
& Spatial (5) & $5.22 \pm 1.56(3.06-6.74)^{\dagger}$ & $1.03 \pm .51(.47-1.84)^{*}$ \\
& Temporal (5) & $4.1 \pm 1.26(2.28-4.61)^{\dagger}$ & $.58 \pm .45(.26-1.37)$ \\
Memory & Immediate recall (10) & $.1 \pm .07[(-.8)-(1.01)]$ & $.36 \pm .14(.08-.5)$ \\
& Semantic memory (2) & $2.35 \pm .34$ & $.74 \pm .36$ \\
Language & Naming (4) & $4.06 \pm 1(3.18-5.31)^{\dagger}$ & $.75 \pm .23(.48-1.03)$ \\
& Repetition (2) & $3.28 \pm .60$ & $.31 \pm .32$ \\
Attention and & Comprehension $\bullet(1)$ & 2.73 & .77 \\
executive func- & Backward calculation (5) & $8.78 \pm 3.58(5.16-14.52)^{\dagger}$ & $6.91 \pm 3.7(3.61-12.85)^{* *}$ \\
tioning & Backward counting (1) & 3.66 & .44 \\
& Abstraction (2) & $4.16 \pm 2.03^{\dagger}$ & $1.19 \pm .18$ \\
\hline
\end{tabular}

Higher values correspond to higher sensitivity and discriminative capability of items. Item difficulty normally ranges from -4 to $4[28,29]$. Items discrimination: "discriminative" $(\geq 1.5)$ or "highly discriminative" ( $\geq 1.7)$ [29]. 'Difficult; *high discrimination; **very high discrimination [28, 29]. ^One out of two items assessing personal orientation was dropped from these analyses as having 0 variance. $\bullet^{\bullet}$ Item originally not dichotomous 


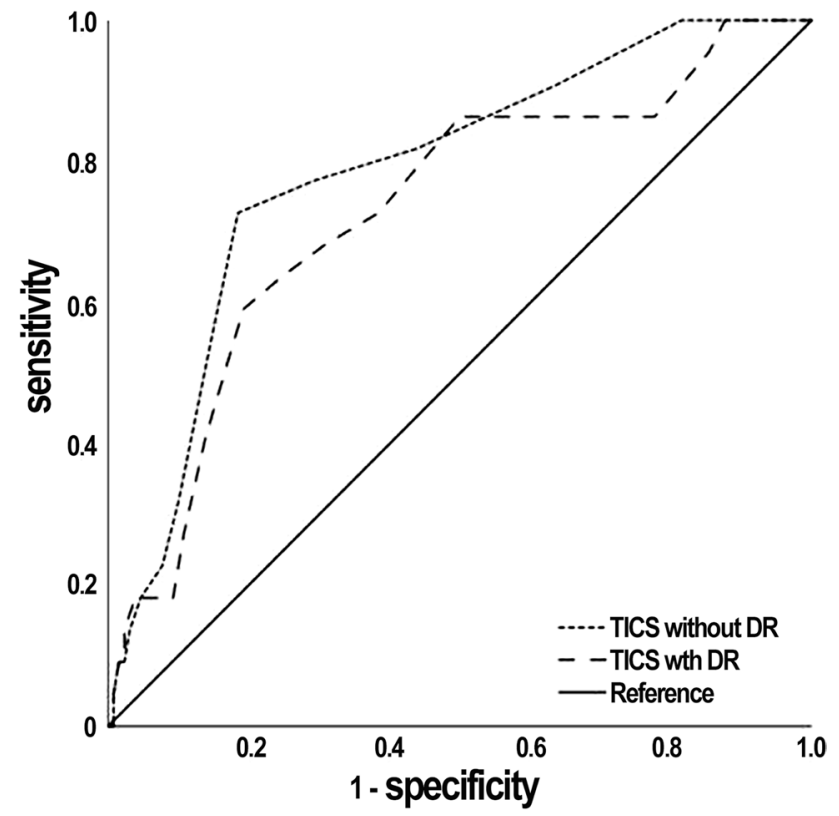

Fig. 2 ROC curves for the TICS with vs. without DR subtest against the Itel-MMSE. The reference measures were a performance above vs. below the 5th percentile on the Itel-MMSE. TICS without DR: $\mathrm{AUC}=.79, \mathrm{SE}=.05,95 \%$ CI $[.69, .89]$; TICS with $\mathrm{DR}: \mathrm{AUC}=.73$, $\mathrm{SE}=.06,95 \% \mathrm{CI}[.61, .85]$

longitudinal studies, a greater reach of underserved [31] or home-locked-down [32] populations, as well as multi-stage prevention campaigns [4]. Furthermore, as minimally relying on physical supports, the TICS might be useful for both bedridden and visually impaired patients [16], as well as to administration in infectious environments [33].

This study relevantly contributes to the literature on of first-level (i.e., cognitive screening) TBCS tools [13], whose utilization is expected to increase with continuous improvement of telehealth care services [34]. Moreover, the robust statistical framework of the present work aligns with the recently underlined need for a greater psychometric rigor when developing/standardizing TBCS instruments [35, 36].

Consistently with previous studies [37], the Italian TICS proved to be a valid measure of general cognitive abilities, thus endorsing its use as a neuropsychological screening test. With this respect, as the highest contribution to its total score was provided by memory items and the orientation subtest yielded the highest correlation with the Itel-MMSE ( $\approx 40 \%$ of whose items assess orientation) [19], the TICS confirms its potential for Alzheimer's spectrum disorders $[10,17]$. However, it should be noted that the off-label DR item did not increase the diagnostic accuracy of the TICS, in line with previous evidence [38]. The present findings thus quantitatively support the adoption of the original TICS format, although the inclusion of the DR task would provide further relevant semiotic information.
Moreover, its excellent inter-rater reliability ensures that, in spite of the remote administration modality, the TICS is minimally dependent of examiners' subjectivity, thus being suitable for usage by several practitioners of similar backgrounds. This last finding is of key importance as TBCS tools have been questioned as possibly being lowly reliable [36].

Item-level features have been shown to be highly relevant to interpretation of TBCS scores [35]. Consistently, those herewith enclosed should lead practitioners to pay particular attention to backwards subtraction items, as proving to be the most informative.

There are some limitations that need to be considered. First, in the present sample, participants aged $\geq 86$ years old are little represented: future investigations should therefore focus on the feasibility of the TICS in the very old population, for which complete normative data often lacks. Second, the region representativeness appears to be moderately biased toward Northern Italy; in this respect it is worth mentioning that the TICS has been shown to be feasible also in Southern Italian individuals in the context of an epidemiological study [39]. Moreover, since the recruitment of participants for the present study occurred during the COVID-19 pandemic, this situation could have, at least to some extent, influenced the present test scores, due to subjective/objective cognitive difficulties observed even in the general, healthy, population during COVI-19-related emergencies [5-7]. However, it should be also noted that, in the present sample, participants had no history of psychiatric/neurological illnesses, hence any potential influence of the pandemic on the findings herewith reported is likely negligible.

There is also an intrinsic limit of the TICS that should be acknowledged, namely, the fact that it requires sufficiently intact hearing as to validly interpret the results. Therefore, the individual hearing status should be thoroughly examined before TICS administration especially in the elders, given the high incidence of audiological decline is elderly [40].

Finally, it should be borne in mind that further investigations are needed on the clinical usability of the Italian TICS in different neurological populations. Indeed, until now, only one study tested its usability in patients with Alzheimer's disease [17].

Supplementary Information The online version contains supplementary material available at https://doi.org/10.1007/s10072-021-05729-7.

Acknowledgements We thank Giunti Psychometrics for providing us with the permission to use data collected within the Italian standardization of the Telephone Interview for Cognitive Status that E.N.A., L.D., N.B., and I.A. are editing on its behalf with Prof. Luigi Ferrucci. We thank Prof. Jason Brandt for providing us with his endorsement toward the project aimed at the Italian standardization of the Telephone Interview for Cognitive Status. We thank Dr. Tiziana Metitieri and Dr. Paolo Caimi for providing us with the permission to use the Italian telephone-based Mini-Mental State Examination. 


\section{Declarations}

Ethical approval This study received ethical approval. Participants provided informed consent.

Conflict of interest E.N.A., L.D., N.B., and I.A. are editing with Prof. Luigi Ferrucci the Italian standardization of the Telephone Interview for Cognitive Status on behalf of Giunti Psychometrics. Giunti Psychometrics holds the copyright for the Italian Telephone Interview for Cognitive Status. I.G. has nothing to declare.

\section{References}

1. Gatz M, Reynolds CA, John R, Johansson B, Mortimer JA, Pedersen NL (2002) Telephone screening to identify potential dementia cases in a population-based sample of older adults. Int Psychogeriatr 14:273-289

2. Smith MM, Tremont G, Ott BR (2009) A review of telephoneadministered screening tests for dementia diagnosis. Am J Alzheimers Dis Other Dementias ${ }^{\circledR}$ 24:58-69

3. Herr M, Ankri J (2013) A critical review of the use of telephone tests to identify cognitive impairment in epidemiology and clinical research. J Telemed Telecare 19:45-54

4. Crooks VC, Clark L, Petitti DB, Chui H, Chiu V (2005) Validation of multi-stage telephone-based identification of cognitive impairment and dementia. BMC Neurol 5:1-8

5. Fiorenzato E, Zabberoni S, Costa A, Cona G (2021) Cognitive and mental health changes and their vulnerability factors related to COVID-19 lockdown in Italy. PLoS One 16:e0246204

6. Maggi G, Baldassarre I, Barbaro A, Cavallo ND, Cropano M, Nappo R, Santangelo G (2021) Mental health status of Italian elderly subjects during and after quarantine for the COVID-19 pandemic: a cross-sectional and longitudinal study. Psychogeriatrics 21:540-551

7. Santangelo G, Baldassarre I, Barbaro A, Cavallo ND, Cropano M, Maggi G, Nappo R, Trojano L, Raimo S (2021) Subjective cognitive failures and their psychological correlates in a large Italian sample during quarantine/self-isolation for COVID-19. Neurol Sci 42:2625-2635

8. Carlew AR, Fatima H, Livingstone JR, Reese C, Lacritz L, Pendergrass C, Bailey KC, Presley C, Mokhtari B, Cullum CM (2020) Cognitive assessment via telephone: a scoping review of instruments. Arch Clin Neuropsychol 35:1215-1233

9. Caze T, Dorsman KA, Carlew AR, Diaz A, Bailey KC (2020) Can you hear me now? Telephone-based teleneuropsychology improves utilization rates in underserved populations. Arch Clin Neuropsychol 35:1234-1239

10. Brandt J, Spencer M, Folstein M (1988) The telephone interview for cognitive status. Neuropsychiatry Neuropsychol Behav Neurol $1: 111-117$

11. Fong TG, Inouye SK (2018) The telephone interview for cognitive status. Cogn Behav Neurol 31:156-157

12. Castanho TC, Amorim L, Zihl J, Palha JA, Sousa N, Santos NC (2014) Telephone-based screening tools for mild cognitive impairment and dementia in aging studies: a review of validated instruments. Frontiers in Aging Neuroscience 6:16

13. Elliott E, Green C, Llewellyn DJ, Quinn TJ (2020) Accuracy of telephone-based cognitive screening tests: systematic review and meta-analysis. Curr Alzheimer Res 17:460-471

14. Seo EH, Lee DY, Kim SG, Kim KW, Kim DH, Jo Kim B, Kim MD, Kim SY, Kim YH, Kim JL, Kim JW (2011) Validity of the telephone interview for cognitive status (TICS) and modified
TICS (TICSm) for mild cognitive imparment (MCI) and dementia screening. Arch Gerontol Geriatr 52:e26-e30

15. Lindgren N, Rinne JO, Palviainen T, Kaprio J, Vuoksimaa E (2019) Prevalence and correlates of dementia and mild cognitive impairment classified with different versions of the modified Telephone Interview for Cognitive Status (TICS-m). Int J Geriatr Psychiatry 34:1883-1891

16. Ferrucci L, Del Lungo I, Guralnik JM, Bandinelli S, Benvenuti E, Salani B, Lamponi M, Ubezio C, Benvenuti F, Baroni A (1998) Is the telephone interview for cognitive status a valid alternative in persons who cannot be evaluated by the Mini Mental State Examination? Aging Clin Exp Res 10(4):332-338

17. Dal Forno G, Chiovenda P, Bressi F, Ferreri F, Grossi E, Brandt J, Rossini PM, Pasqualetti P (2006) Use of an Italian version of the telephone interview for cognitive status in Alzheimer's disease. Int J Geriatr Psychiatry 21:126-133

18. Metitieri T, Geroldi C, Pezzini A, Frisoni GB, Bianchetti A, Trabucchi M (2001) The Itel-MMSE: an Italian telephone version of the Mini-Mental State Examination. Int J Geriatr Psychiatry $16: 166-167$

19. Vanacore N, De Carolis A, Sepe-Monti M, Bomboi G, Stazi A, Bianchetti A, Giubilei F (2006) Validity of the Italian telephone version of the Mini-Mental State Examination in the elderly healthy population. Acta Neurol Belg 106:132-136

20. R Core Team (2021) R: a language and environment for statistical computing. (Version 4.1.0) [Computer software]. Retrieved from https://cran.r-project.org

21. IBM Corp. (2021). IBM SPSS Statistics for Windows, version 27.0. Armonk, NY: IBM Corp.

22. StataCorp (2019) Stata statistical software: release 16. StataCorp LLC, College Station

23. Champely, S. (2020). pwr: basic functions for power analysis $(\mathrm{R}$ package version 1.3.0) [Computer software]. The Comprehensive R Archive Network. Available from https://CRAN.R-project.org/ package $=$ pwr

24. Kim HY (2013) Statistical notes for clinical researchers: assessing normal distribution (2) using skewness and kurtosis. Restor Dent Endod 38:52-54

25. Rizopoulos D (2006) ltm: an R package for latent variable modelling and item response theory analyses. J Stat Softw 17:1-25

26. Baylor C, Hula W, Donovan NJ, Doyle PJ, Kendall D, Yorkston K (2011) An introduction to item response theory and Rasch models for speech-language pathologists. Am J Speech Lang Pathol 20:243-259

27. Chalmers RP (2012) mirt: a multidimensional item response theory package for the R environment. J Stat Softw 486:1-29

28. Hambleton RK, Swaminathan H, Rogers HJ (1991) Fundamentals of item response theory. Sage Publications, California, pp 7-31

29. Baker FB, Kim SH (2017) The basics of item response theory using R. Springer, New York, pp 17-34

30. Petersen RC, Thomas RG, Grundman M, Bennett D, Doody R, Ferris S, Galasko D, Jin S, Kaye J, Levey A, Pfeiffer E, Sano M, van Dyck CH, Thal LJ (2005) Vitamin E and donepezil for the treatment of mild cognitive impairment. N Engl J Med 352:2379-2388

31. De Cola MC, Triglia G, Camera M, Corallo F, Di Cara M, Bramanti P, Lo Buono V (2020) Effect of neurological screening on early dementia detection in southern Italy. J Int Med Res 48:1-11

32. Soldati AB, Almeida C, Lima M, Araujo A, Araujo-Leite MA, Silva MTT (2021) Telephone screening of cognitive status (TICS) in severe COVID-19 patients: utility in the era of social isolation. Eneurologicalsci 22:100332

33. Trapp W, Röder S, Heid A, Billman P, Daiber S, Hajak G (2021) Sensitivity and specificity of the Bamberg Dementia Screening Test's (BDST) full and short versions: brief screening instruments 
for geriatric patients that are suitable for infectious environments. BMC Med 19:1-13

34. Kwan RYC, Lai CKY (2013) Can smartphones enhance telephone-based cognitive assessment (TBCA)? Int J Environ Res Public Health 10:7110-7125

35. Booth T, Murray A, Muniz-Terrera G (2021) Are we measuring the same thing? Psychometric and research considerations when adopting new testing modes in the time of COVID-19. Alzheimers Dement 17:251-254

36. Hunter MB, Jenkins N, Dolan C, Pullen H, Ritchie C, MunizTerrera G (2021) Reliability of telephone and videoconference methods of cognitive assessment in older adults with and without dementia. J Alzheimer's Dis 4:1625-1647

37. Van Den Berg E, Ruis C, Biessels GJ, Kappelle LJ, van Zandvoort MJ (2012) The Telephone Interview for Cognitive Status (modified): relation with a comprehensive neuropsychological assessment. J Clin Exp Neuropsychol 34:598-605
38. Yaari R, Fleisher AS, Gamst AC, Bagwell VP, Thal LJ (2006) Utility of the telephone interview for cognitive status for enrollment in clinical trials. Alzheimers Dement 2:104-109

39. Simeon V, Chiodini P, Mattiello A, Sieri S, Panico C, Brighenti F, Krogh V, Panico S (2015) Dietary glycemic load and risk of cognitive impairment in women: findings from the EPIC-Naples cohort. Eur J Epidemiol 30:425-433

40. Quaranta A, Assennato G, Sallustio V (1996) Epidemiology of hearing problems among adults in Italy. Scand Audiol Suppl 42:9-13

Publisher's note Springer Nature remains neutral with regard to jurisdictional claims in published maps and institutional affiliations. 